\section{Ampullary adenocarcinoma with osteoclast giant cells}

Periampullary neoplasms collectively include carcinomasarising from the duodenum, ampulla of Vater, distal part of the common bile duct and head of pancreas. ${ }^{1}$ Ampullary carcinoma constitutes $7 \%$ of all peri-ampullary carcinomas with adenocarcinoma being the commonest histologic type. ${ }^{2}$ Osteoclast giant cells (OGCs) have been reported to be seen in various malignanciesarising from the pancreas, breast and rarely the liver, kidney, urinary bladder, gall bladder and the endometrium. ${ }^{3,4}$ Ampullary adenocarcinoma with OGCs is an extremely rare entity and has only sparsely been described in literature till date. ${ }^{5}$

\section{Case Report}

A 53 year-old post-menopausal female presented with yellowish discoloration of the eyes, clay-colored stools and high-colored urine for 6 months. She reported a loss of appetite, an unquantified loss of weight, occasional pain abdomen radiating to the back and multiple episodes of cholangitis. She had a history of endoscopic retrograde cholangio-pancreatography (ERCP) followed by laparoscopic cholecystectomy 5 months back at a private hospital. Subsequently, in view of the recurrent cholangitis, repeat ERCP and stent exchange was performed thrice before she was referred to our hospital. Computed tomography scan of the abdomen showeda mass in the peri-ampullary region causing dilatation of the common bile duct and the pancreatic duct along with atrophy of the body and tail of pancreas. An MRI showed a hyperintenseperi-ampullarylesion on T1-weighted images. A diagnosis of adenocarcinoma was made following an endoscopic ultrasound-guided biopsy. The patient subsequently underwent a pylorus-resectingpancreaticoduodenectomyalong with appropriate lymphadenectomy. A grey-white ulcero-infiltrative tumour measuring $4 \mathrm{~cm} \times 3 \mathrm{~cm} \times 1.8 \mathrm{~cm}$ was identified in the ampullary and periampullary region of the specimen (Figure 1). Microscopic evaluation revealed a tumour composed of variably sized and closely packed glands lined by tumour cells exhibiting nuclear enlargement with pleomorphism

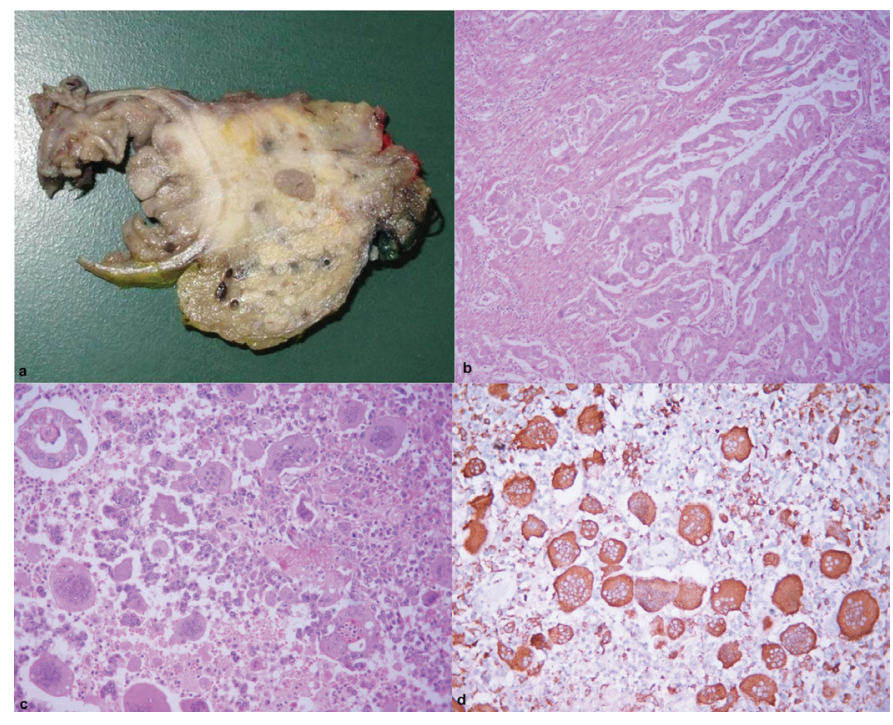

Figure 1(a): Gross examination showing an ulceroinfiltrative grey-white ampullary lesion (b): Closely packed variably sized and shaped glands lined by atypical cells, H\&E X200. (c): Areas rich in osteoclast giant cells and interspersed mononuclear stromal cells, H\&E X200. (d): Osteoclast giant cells and mononuclear stromal cells stain positive for CD68, X200.

and at places, prominent nucleoli were seen. Mucin was also seen in the glandular lumina. The tumour showed areas rich in OGCs and interspersed mononuclear stromal cells. The adenocarcinoma areas were intimately admixed with OGC rich areas. Immunohistochemically, the tumor cells showed positivity for cytokeratin 7 (CK7) and were negative for CD68 while the OGCs and mononuclear stromal cells were positive for CD68 and negative for CK7. The tumor infiltrated the duodenal wall and superficially involved the adjacent pancreas. The non-involved pancreas showed features of chronic pancreatitis. Perineural and lympho-vascular invasion were seen. The dissected lymphnodes showed tumour metastases. However, the metastases was composed of the adenocarcinoma component only. A final diagnosis of moderately differentiated adenocarcinoma with osteoclast giant cells was offered. The pathological stage was T3N1M0, IIB. The postoperative course was uneventful except for mild superficial surgical site infection. However, the patient succumbed to death 4-5 months post-surgery. 


\section{Discussion}

Ampullary carcinomas are rare tumorsthat account for approximately $0.5 \%$ of all gastrointestinal malignancies. Adenocarcinoma $(65 \%)$ is the most common histologic type usually showing either an intestinal or pancreato-biliary type of differentiation. The less common histological subtypes include mucinous, signet ring cell, neuroendocrine and undifferentiated carcinomas. ${ }^{6}$ Westgaard et $\mathrm{al}^{7}$ demonstrated that histological subtyping has prognostic implications with the intestinal subtype showing a better prognosis compared to the pancreaticobiliary type. Other prognostic factors include tumor size, grade, lymph node status, perineural invasion, vascular invasion and locoregional infiltration. ${ }^{7}$

Giant cell tumours (GCTs) can be seen in a wide variety of sites including the pancreas, breast, thyroid, parotid, gall bladder, colon, skin, orbit, kidney, heart and soft tissue. In the pancreas, three kinds of GCTs are knownnamely osteoclastic, pleomorphic and mixed types. Subtyping is considered important as the osteoclastic subtype has a better prognosis than the pleomorphic type. ${ }^{3}$ The exact mechanism of OGCs formation in tumours is not known. The histogenesisis controversial, with suggestions of both epithelial and mesenchymal origin. The pure osteoclastic subtype that resembles giant cell tumor of bone is probably mesenchymal in origin as it is composed of OGCs and mononuclear stromal cells only and lacks epithelial elements. The OGCs and stromal cells stain positive forvimentin and CD68 while are non-reactive for cytokeratin, thereby, confirming a mesenchymal origin. In contrast, the pleomorphic subtype, which does not reveal any particular morphologic epithelial differentiation, shows positivity for cytokeratin and negativity for CD68 and vimentin favouring an epithelial origin. ${ }^{3,8}$ However, there seems to be an overlap between the above discussed types as a mixed subtype also exists. The mixed subtype can have well defined carcinomatous areas as well as a stromal component rich in OGCs. Our case was a mixed one as it had areas of adenocarcinoma and other areas rich in OGCs.

Shishido-Hare et $\mathrm{al}^{4}$ demonstrated in breast cancer that the appearance of OGCs in stroma is due to the pro-tumoral differentiation of macrophages as aresult of the hypervascular micro-environment induced by cancer rather than an antitumoral immunological reaction. Presence of OGCs in breast carcinomas has been suggested to impart a less favorable outcome. ${ }^{4}$ The origin and role of OGCs in various tumors is, thus, controversial and the behavior of these tumours is also widely variable. The presence of components other than OGCs such as glandular, pleomorphic and stromal does seem to greatly influence the behavior of giant cell rich tumors. Our case showed an aggressive behavior despite the absence of marked pleomorphism which is most likely due to the presence of the conventional glandular component, perineural invasion, lympho-vascular invasion and lymph nodal adenocarcinomatous metastatic deposits.

Surgical resection is considered the first line of treatmentand the role of adjuvant therapy is unclear. Radiotherapy has been tried in view of the existing knowledge regardingthe radio-sensitivity of GCTs of the bone. Chemotherapeutic agents like gemcitabine may be used in cases of disseminated disease or incomplete resections. $^{8}$

To conclude, ampullary adenocarcinoma with OGCs is a rare entity and shows an aggressive behavior, thus, suggesting the need for intensive multimodality management.

TARUN KUMAR $^{1}$
MAITRAYEE ROY $^{1}$
RAJNI YADAV $^{1}$
HEMANT JAIN $^{2}$
SUJOY PAL $^{2}$
PRASENJIT DAS

${ }^{1}$ Department of Pathology, ${ }^{2}$ Department of Gastrointestinal Surgery, All India Institute of Medical Sciences, New Delhi,

India.

Correspondence: Dr Rajni Yadav Email:drrajniyadav@gmail.com

\section{References}

1. Kimura W, Futakawa N, Zhao B. Neoplastic diseases of the papilla of Vater. J Hepatobiliary Pancreat Surg. 2004;11:223-31. 
2. O’Connell JB, Maggard MA, Manunga J, Tomlinson JS, Reber HA, Ko CY et al. Survival after resection of ampullary carcinoma: a national population-based study. Ann Surg Oncol. 2008;15:1820-7.

3. Temesgen WM, Wachtel M, Dissanaike S. Osteoclasticgiant cell tumor of the pancreas. Int J Surg Case Rep. 2014;5:175-9.

4. Shishido-Hara Y, Kurata A, Fujiwara M, Itoh H, Imoto S, Kamma H. Two cases of breast carcinoma with osteoclastic giant cells: Are the osteoclastic giant cells pro-tumoural differentiation of macrophages? Diagn Pathol. 2010;5:55.

5. Matsuzawa G, Shirabe K, Gion T, Tsujita E, Ooya M, Kajiyama K. Surgically resected undifferentiated carcinoma with osteoclast-like giant cells of the periampullary region involving the orifice of the papilla of Vater: Report of a case. Surg Today. 2010;40:376-9.

6. Fischer HP, Zhou H. Pathogenesis of carcinoma of the papilla of Vater. J Hepatobiliary Pancreat Surg. 2004;11:301-9.

7. Westgaard A, Tafjord S, Farstad IN, Cvancarova M, Eide TJ, Mathison $\mathrm{O}$ et al. Pancreatobiliary versus intestinal histologic type of differentiation is an independent prognostic factor in resected periampullary adenocarcinoma. BMC Cancer. 2008;8:170-4.

8. Moore JC, Bentz JS, Hilden K, Adler DG. Osteoclastic and pleomorphic giant cell tumors of the pancreas: a review of clinical, endoscopic, and pathologic features. World J Gastrointest Endosc. 2010;2:15-9.

\section{Median arcuate ligament syndrome (MALS) in rural Prakasam district of Andhra Pradesh}

Harjola $^{1}$ was the first surgeon who described the celiac axis compression syndrome or Median Arcuate Ligament syndrome in 1963. Subsequently, J David Dunbar and Samuel Marable ${ }^{2}$ also spoke about the same in 1965 and so, it is called the Harjola-Marable syndrome. The median arcuate ligament is formed near the 12th thoracic vertebra and usually comes into contact with the aorta above the branch point of the celiac artery. ${ }^{3}$ Sometimes, the median arcuate ligament passes in front of the celiac artery, thus compressing it and presents with the triad of abdominal pain, weight loss and vomiting.

One theory proposes that compression of the celiac artery causes is chemiaor decreased blood flow to the abdominal organs, thus leading to pain. Another hypothesis that there is compression not only of the celiac artery but also of the celiac ganglia. ${ }^{4}$ It typically occurs in young patients (20 to 40 years of age) and is more common in thin women. ${ }^{5}$ We report one such case which was diagnosed using CT angiography and was managed effectively using a holistic approach by a team of doctors.

\section{Case Report}

A 21-year old unmarried rural young male had consulted in multiple hospitals for postprandial pain. After a threeyear history of intermittent abdominal pain and decreased appetite, he presented to us with recurrent episodes of epigastric pain of moderate to severe intensity associated with vomiting and constipation. He had been suffering similar complaints at least 5 to 6 times in a year with each episode is lasting for 5 to 7 days and responding to supportive treatment. The frequency had increased in the last one year. He is the second sibling of a consanguineous family. No significant health problems were noticed in his family members. He is neither an alcoholic nor a smoker and has lost $8 \mathrm{kgs}$ in 2 year. There is no history of neurological symptoms, skin manifestations, urinary symptoms and per rectal bleeding. Per abdomen was soft with mild tenderness observed in the epigastric region.

Complete blood count (CBC), amylase, lipase, liver function tests, renal function tests, fasting blood sugar, bleeding time and clotting time were normal. This patient was negative for HBsAg, HCV and HIV infections. Peripheral blood smear for malarial parasite, Widal test for Salmonella and serum levels of electrolytes like sodium, potassium and chloride were also normal.

Ultrasound abdomen and X-ray barium did not reveal any significant problem. Gastritis was noticed on upper GI endoscopy while colonoscopy was normal. 